\title{
PAVEMENT DIAGNOSIS AS INTEGRANT OF THE PAVEMENT MANAGEMENT SYSTEM
}

The paper integrates the most important scientific information of the authors in the field of diagnostics of road pavements. Basic attributes of pavement serviceability (evenness, skid resistance) and pavement bearing capacity are the core of the paper. The results of simulations of dynamic systems, outputs of the models developed at the authors place, the correlations to the world established parameters of evenness and skid resistance are presented in the paper. Objective scientific outputs have formed an effectual base for development the Slovak Pavement Management System (PMS). The principles of the Slovak PMS are described.

\section{Introduction}

The Slovak Pavement Management System (PMS) [10] is a tool for effective dividing of budget for the management of road rehabilitation. The system includes processes for effective maintenance, repairs and renewal of road surfaces and structures. The processes are based on diagnostics of the pavement surface parameters (serviceability level of pavement) and bearing capacity. These parameters input into PMS as follows:

- Surface failures [2] - input by Index of surface deterioration (ISD) describing ratio of failures area to surface area. Evaluation criteria include five levels of quality (from excellent to emergency).

- Longitudinal unevenness [2] - input by International Roughness Index (IRI) describing longitudinal unevenness quality in five levels.

- Transversal unevenness [2] - input by Ruts depth represents the transversal unevenness. The quality is evaluated in five levels scale as for longitudinal unevenness.

- Skid resistance [2] - input by Skid Resistance Index (SRI) describing skid resistance in relation to microtexture and macrotexture quality. The methodology of quality evaluation uses three levels of assessment.

- Bearing capacity uses deflection bowls measured by FWD Kuab as an input for the program CANUV [1] that gives the possibility to determine modulus of pavement layers to calculate residual pavement life and overlay.

The technology of pavement rehabilitation is chosen according to all the parameters. Evaluation of these variable parameters (except surface failures) in scope of the Slovak PMS and basic principles of PMS are presented in the next part of the paper.

\section{Longitudinal unevenness}

The discretionary evaluated road sections, which are homogenous from the point of view of construction and degradation conditions, can be evaluated through the medium theory of stationary random process. This type of random process can be best characterized by a correlation function or power spectral density (PSD). The correlation function $K_{h}(\lambda)$ for this type of process is expressed in linear domain by equation

$$
\begin{aligned}
& K_{h}(\lambda)=\int_{-\infty}^{\infty} \int_{-\infty}^{\infty}\left[h_{1}(l)-E_{h}\right] \cdot\left[h_{2}(l-\lambda)-E_{h}\right] . \\
& f_{2}\left(h_{1}, h_{2}\right) d h_{1} \cdot d h_{2}
\end{aligned}
$$

where: $\lambda \quad-$ linear lag $[\mathrm{m}]$,

$E_{h} \quad-$ expected value of stochastic unevenness; $E_{h}=0$,

$h(l) \quad$ - stochastic unevenness,

$f_{2}\left(h_{1}, h_{2}\right)$ - combination density of expectation.

Stochastic unevenness is computed as a difference between a real and theoretical profile. In our case we must identify elevations of longitudinal profile per $0.25 \mathrm{~m}$ and longitudinal unevenness are evaluated through the standardized correlation function $\rho_{h}(\lambda)$ (left hand side of Fig. 1).

For the purpose of unevenness assessment it is more appropriate to use power spectral density (PSD) $S_{h}(\Omega)$ (right hand side of Fig. 1), which can be expressed from the correlation function by means of Wiener Chinchine equation:

$$
S_{h}(\Omega)=2 / \pi \cdot \int_{0}^{\infty} K_{h}(\lambda) \cdot \cos (\Omega \lambda) \cdot d \lambda
$$

where: $D_{h}$ - dispersion of an stochastic unevenness $\left[\mathrm{m}^{2}\right]$,

$\Omega$ - angular spatial frequency [rad. $\mathrm{m}^{-1}$ ],

$$
\Omega=2 \cdot \pi / L
$$

\footnotetext{
* Jan Celko, Martin Decky, Jozef Komacka, Matus Kovac

Department of Highway Engineering, Faculty of Civil Engineering, University of Zilina, Slovakia, E-mail: jan.celko@fstav.uniza.sk
} 
$\rho_{h}(\lambda)=K_{h}(\lambda) D_{h}$
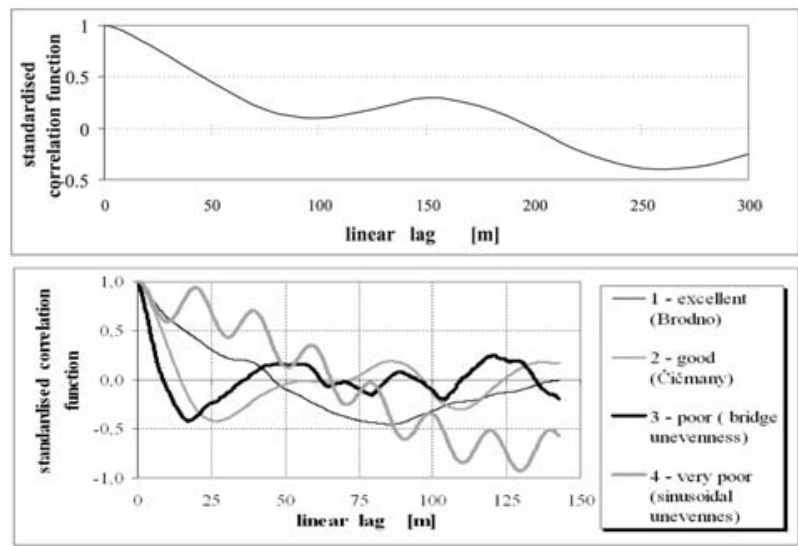
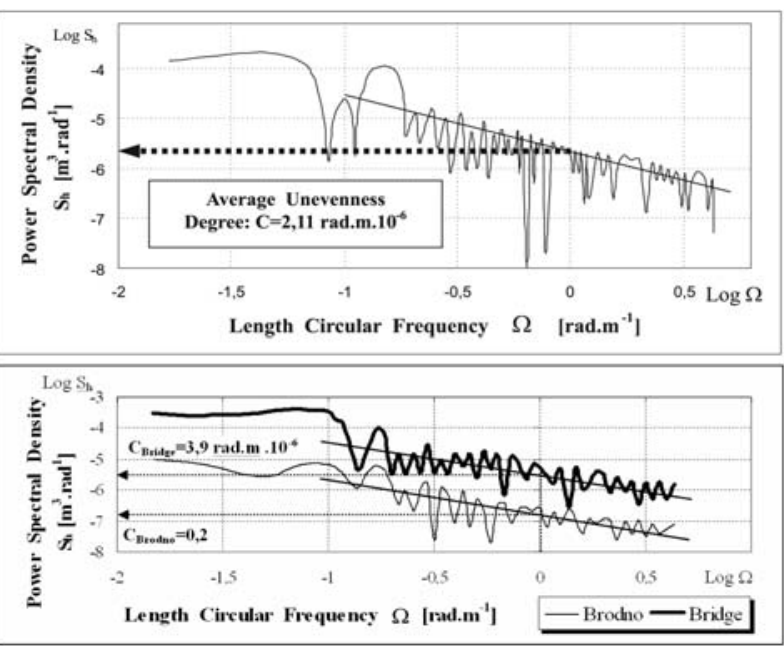

Fig. 1 Standardized correlation function and PSD of stochastic unevenness
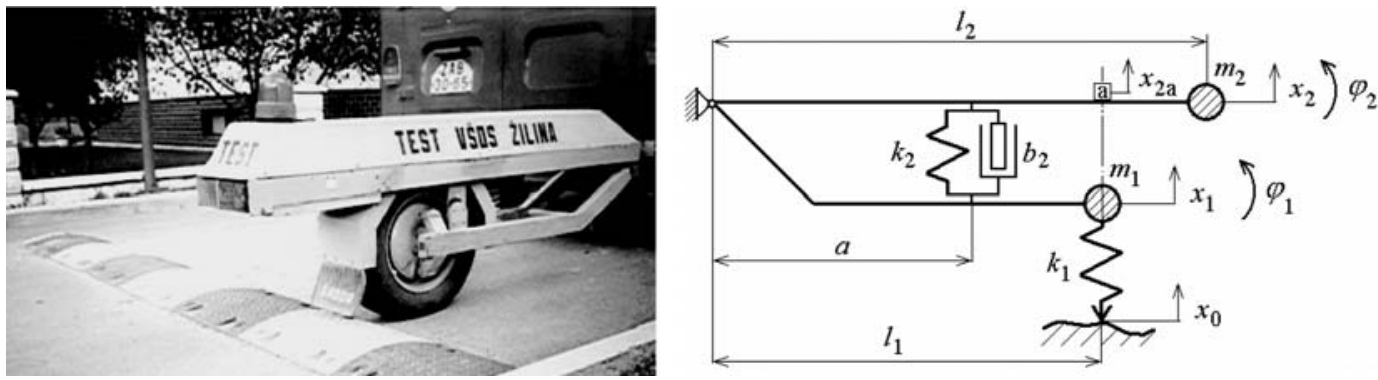

Fig. 2 Single-Wheel Vehicle of the University of Žilina

The measuring set called the Single-Wheel Vehicle of the University of Žilina (Slovak abbr. JP VŠDS) was designed on the DMS (double-mass measuring set) principle. This equipment represents a model of a quarter of the passenger vehicle and its basic parts are presented in Fig. 2.

For vertical vibrations of a quarter car model around the static equilibrium positions we obtain the following equations of motion The equations of motion for small vibrations are given by [3].

The vertical displacement (velocity, acceleration) of mass $\mathrm{m}^{2}$ can be expressed as a function of geometry and displacement (velocity, acceleration) of accelerometer as follows:

$$
x_{2}=\left(l_{2} / l_{1}\right) \cdot x_{2 a}, \dot{x}_{2}=\left(l_{2} / l_{1}\right) \cdot \dot{x}_{2 a}, \ddot{x}_{2}=\left(l_{2} / l_{1}\right) \cdot \ddot{x}_{2 a}
$$

After substituting (5) to equations in [3] we can rewrite these relations as:

$$
\begin{aligned}
& m_{1} \ddot{x}_{1}+b_{2} \frac{a^{2}}{l_{1}^{2}}\left(\dot{x}_{1}-\dot{x}_{2 a}\right)+k_{2} \frac{a^{2}}{l_{1}^{2}}\left(x_{1}-x_{2 a}\right)+ \\
& +k_{1} x_{1}=k_{1} x_{0}
\end{aligned}
$$

$$
m_{2} \ddot{x}_{2 a}+b_{2} \frac{a^{2}}{l_{2}^{2}}\left(\dot{x}_{2 a}-\dot{x}_{1}\right)+k_{2} \frac{a^{2}}{l_{2}^{2}}\left(x_{2 a}-x_{1}\right)=0 .
$$

The unevenness degree $C$ [rad.m. $10^{-6}$ ] of an evaluated road section is expressed from the basic relation [9] that was modified for our mode of unevenness identification by JP VŠDS.

$$
C=\frac{D_{y}}{I \cdot \frac{1}{N} \sum_{i=1}^{n} v_{i}}
$$

where: $D_{y}$ - dispersion of sprung mass acceleration - left hand side of Fig. $3\left[\mathrm{~m}^{2} . \mathrm{s}^{-4}\right]$,

$I$ - parameter of dynamic transfer $\left[\mathrm{rad}^{-1} . \mathrm{s}^{-3}\right]$,

$C$ - unevenness degree - right hand side of Fig. 3 [rad.m],

$v_{i}$ - digital values of a measured velocity - right hand side of Fig. 3 [m.s ${ }^{-1}$.

The correlations between unevenness degree $C$ and IRI (International Roughness Index) have been determined for a consideration of theoretical dynamical response of RQCS (Reference Quarter Car Simulation) [8] and experimental measurements (Fig. 4). 

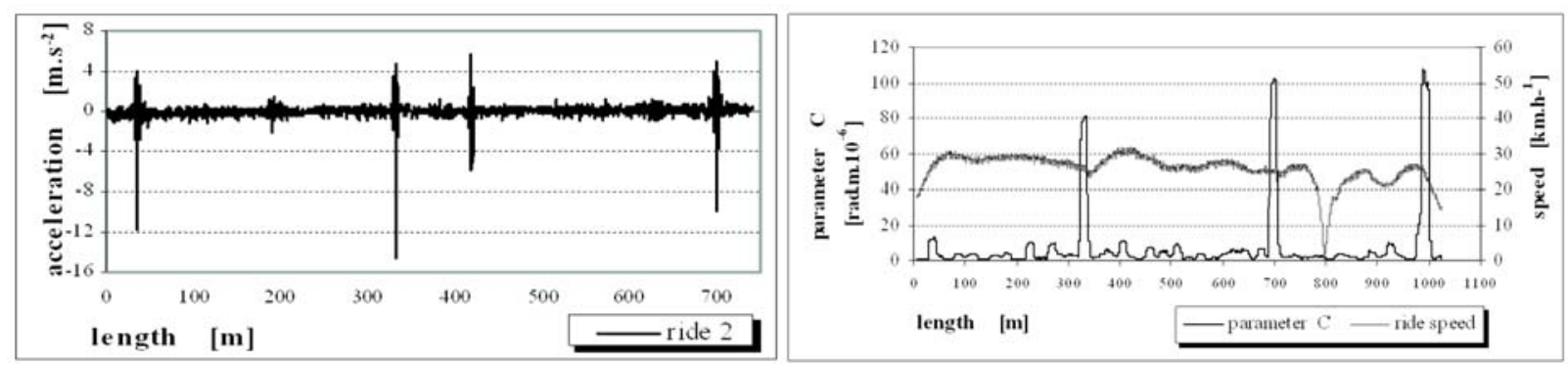

Fig. 3 Vertical acceleration, ride speed and parameter C evaluated by JP VSDS
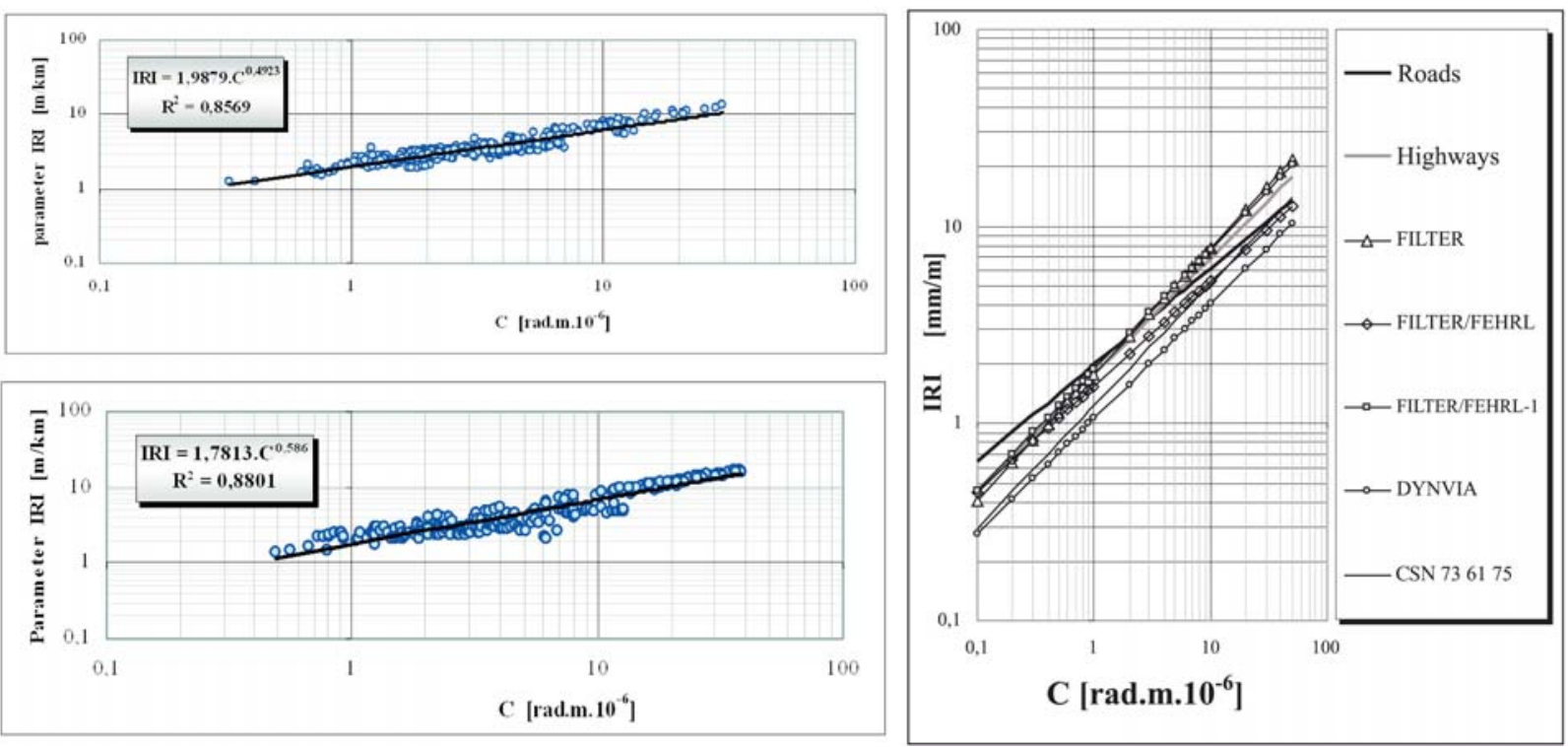

Fig. 4 Comparison of Slovak and World-wide correlation dependences between unevenness degree C and IRI

The comparison of our correlation dependences (roads - ride speed of $90 \mathrm{kph}$ and highways of $130 \mathrm{kph}$ ) with results of the Second International PIARC-WRA experiment (including FILTER programme) [3], [4], [11] can be seen in Fig. 4.

\section{Skid resistance}

Pavement skid resistance was in the past evaluated by different parameters. The parameters were oriented to macrotexture qualify or to detection of skid resistance of pavement surface. At present there are a lot of efforts to unify the measurement evaluation to acquire a common index which is necessary for objective characterization of pavement quality in dependence on its skid resistance properties.

SRI (Skid Resistance Index) is a new methodology mostly used for measurement and evaluation of pavement skid resistance. The Slovak Road Administration devices (SKIDDOMETER BV 11 and PROFILOGRPH GE) are used in Slovakia. Following inputs for estimation of SRI are needed:
- Friction evaluated by Mu from device SKIDDOMETER BV11,

- Operating speed (v) by SKIDDOMETER BV 11,

- Macrotexture evaluated by MPD from device PROFILOGRAPH GE, or by MTD from the volumetric sand patch method.

Taking into account the results of the international harmonization experiment which was focused on measurement and evaluation of pavement skid resistance the estimation of the Skid resistance index (SRI) should be computed by the following equation

$$
S R I=A+B \cdot F R S \cdot e^{\left(S-S_{R}\right) / S_{0}}
$$

where: $A, B$ - particular parameters for each device,

$F R S$ - reported friction value from the test device (an average value for a chosen interval),

$S_{r} \quad$ - reference slip speed determined to $30 \mathrm{kph}$,

$S_{0} \quad$ - speed parameter linearly related to the result of a macrotexture measurement,

$S$ - slip speed (relative speed between the tire and the travelled surface at the contact area. 
Slip speed is calculated as

$$
S=v^{*} \text { slip ratio }
$$

where: $v \quad$ - operating speed of the device [kph],

slip ratio - quotient of the slip speed divided by operating speed (for SKIDDOMETER BV11 is $\left.S=0.17^{*} v\right)$.

The speed parameter is calculated from the equation

$$
S_{0}=a+b \cdot M P D
$$

where: MPD - Mean Texture Depth from the device PROFILOGRAPH GE [mm],

$a, b$ - particular parameters for each device.

The parameters $\mathrm{a}, \mathrm{b}$ were determined from comparative measurements performed in Slovakia with a particular device to achieve the best correlation with the sand patch test method (MTD). Figure 5 shows the correlation between MTD and MPD measured by PROFILOGRAPH GE. On the base of the determined relation the value $S_{0}(12)$ is calculated. The value is necessary for estimation of SRI (13).

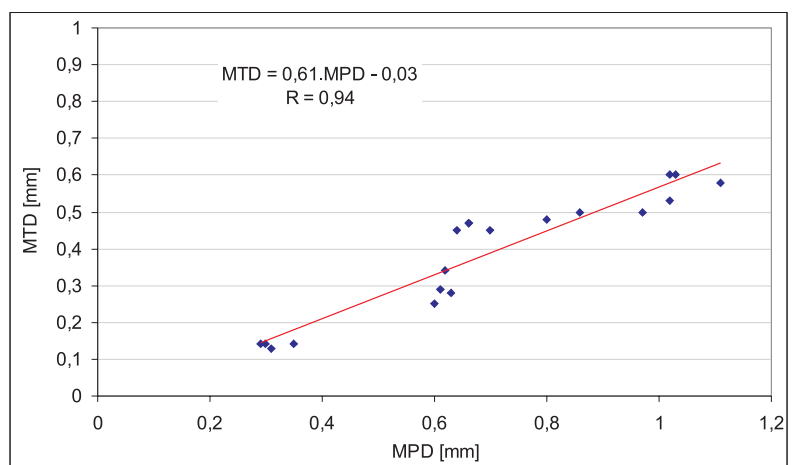

Fig. 5 MPD by PROFILOGRAPH GE versus MTD by Sand Patch Method

Final relations for estimation of SRI for devices which have been used in Slovakia could be defined as follows:

$$
S_{0}=68.16 \cdot M P D-15.0
$$

$$
S R I=0.101+0.78 * M u * e^{-(S-30) / S_{0}}
$$

These equations are defined for the estimation of the Skid Resistance Index for the SKIDDOMETER BV11. The SRI shows a good correlation with other pavement skid resistance measurement devices with an operating speed allowance. Figure 6 shows the correlation between the British Pendulum Tester which is one of the mostly used devices in the world.

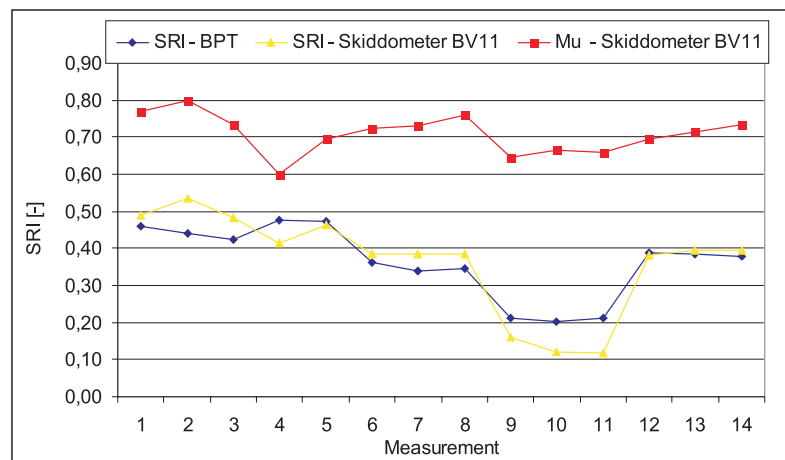

Fig. 6 Relation between SRI and Mu by SKIDDOMETER BVI1 and SRI by British Pendulum Tester

\section{Pavement bearing capacity}

Diagnostics and evaluation of pavement bearing capacity is the important element in the majority of the Pavement Management Systems. Non-destructive testing methods based on the measuring of pavement surface deflection are the most extended in this field. Measured values of deflections are used for an analysis of pavement structure response to an applied load. Characteristics necessary for the pavement evaluation are derived from deflections using some theoretical assumptions. Majority of the used methods replace the real pavement structure by the physical model of linear elastic halfspace or linear elastic multi layered half-space. Measured deflections are then considered as vertical deformations of the physical pavement model. These assumptions give the possibility to determine the surface modulus of pavement structure or the modulus of individual pavement layers from the back calculation.

\subsection{Evaluation according to surface modulus}

Basic theory of linear elastic half space was used for calculation of the pavement surface modulus. The model of static loading of linear elastic half-space by a vertical uniform load was used and dynamic effect of the applied load was neglected. It was assumed that vertical deformations (deflections) of the pavement surface correspond with vertical deformations caused by a static load. Then, within the ambit of a linear elastic half-space theory, when the load is distributed through the elastic load plate of diagnostics equipment the surface modulus of pavement in the centre of load can be calculated from the equation

$$
E=\frac{2 \cdot\left(1-\mu^{2}\right) \cdot p_{0} \cdot a^{2}}{w(0)_{z=0}}
$$

and the pavement surface modulus at the distance " $r$ " from the centre of load (but $r>a$ ) as follows

$$
E_{(r)}=\frac{\left(1-\mu^{2}\right) \cdot p_{0} \cdot a^{2}}{w(r)_{z=0} \cdot r}
$$


where: $\mu$ - Poisson's ratio,

$p_{o}$ - contact pressure [MPa],

$a$ - radius of loading plate $[\mathrm{m}]$,

$r \quad$ - distance from the centre of load [m],

$w(0) z=0-$ deflection of pavement surface at the axis of load $[\mathrm{m}]$.

The surface modulus of pavement calculated according to (14) and (15) is the simplest characteristic of the pavement bearing capacity and it is very often used for evaluation of the pavement structure in terms of its mechanical efficiency and homogeneity. The same principle was used for determination of a criterion for the pavement bearing capacity evaluation in Slovakia at the network level [9].

\subsection{Computer program for evaluation}

The surface modulus is not sufficient for evaluation at the project level. In this case it is necessary to determine the modulus of individual pavement layers. For this purpose the program CANUV [9] was developed at the Highway Engineering Department of Civil Engineering Faculty. Basic input data are deflection bowls measured by the Falling Weight Deflectometer KUAB. The program uses a back-calculation procedure to determine the modulus of tree main pavement layers (asphalt layers, subbase, subgrade). The principle of back calculation of the modulus is to fit a measured deflection bowl to a theoretical bowl calculated for a set of layer modulus. The theory of an elastic multi-layered system is used for the calculation of the theoretical bowls (deflections). The back-calculation procedure finishes when the following criteria are met:

$$
\begin{aligned}
& \frac{w_{m, 1500}-w_{c, 1500}}{w_{m, 1500}} \times 100<2 \\
& \frac{\left(w_{m, 0}-w_{m, 300}\right)-\left(w_{c, 0}-w_{c, 300}\right)}{\left(w_{m, 0}-w_{m, 300}\right)} \times 100<1 \\
& \frac{w_{m, 900}-w_{c, 900}}{w_{m, 900}} \times 100<4
\end{aligned}
$$

where: $w_{m, i}$ - measured deflection in distance " $i$ " from the centre of load $[\mu \mathrm{m}]$,

$w_{c, i}$ - calculated deflection in distance " $i$ " from the centre of load $[\mu \mathrm{m}]$.

After the modulus back-calculation the reliability of the pavement structure is evaluated. The relation between the bending tensile stress and the material strength with regard to the material fatigue is applied in this process for bound pavement layers

$$
S=\sigma /\left(S_{n} \cdot R\right)
$$

and the pavement subgrade reliability evaluation is calculated from the formula

$$
S=\sigma_{z} / \sigma_{z, m}
$$

where: $S_{n} \quad$ - material fatigue coefficient $\left(S_{n}=a-b \cdot \log N\right)$,

$R$ - tensile-bending strength of the material in the critical layer [MPa],

$\sigma \quad$ - bending tensile stress at the bottom of the layer [MPa].

Provided that $S=1$ the residual performance of pavement bound layers is calculated as

$$
\log N_{b}=(a \cdot R-\sigma) / b \cdot R
$$

where: $N_{b}$ - residual number of standard axles for bound layer, $a, b$ - fatigue coefficients of bound layer

and the residual performance of subgrade is calculated as

$$
\log N_{S}=\frac{0.00346 \times E_{S}-\sigma_{z}}{0.7 \cdot \sigma_{z}}
$$

where: $N_{s}$ - residual number of standard axles for subgrade,

$z \quad$ - computed vertical stress on the top of subgrade [MPa],

$E_{s} \quad$ - modulus of subgrade determined by back calculation [MPa].

The lowest computed value from $N_{b}$ and $N_{s}$ determines the "critical layer" of the pavement. The residual performance in standard axles in future years is calculated for this layer from

$$
N_{R}=N_{i}-\sum_{r=1}^{n} N_{n} \times \delta_{i}
$$

where: $N_{R}$ - residual performance of critical layer in the year " $\mathrm{r}$ ",

$N_{i} \quad$ - total residual performance of the critical pavement layer,

$N_{n}$ - annual number of standard axles in a design section,

$\delta_{i} \quad$ - annual increase of the traffic in a design section [\%].

For the first $N_{R}<0$ residual life of pavement (RL) in the years is calculated from the value of index " $\mathrm{r}$ " as $R L=r-1$. The pavement reliability in term of bearing capacity is classified according to the value of residual life.

\section{Slovak Pavement Management System}

The most important benefit of PMS is a more objective distribution of financial resources based on an economic analysis, the construction and user costs included. The Slovak PMS works at two levels:

- the road network level solving the selection of sections for rehabilitation, budget requirements and distribution of financial resources;

- project level solving a proposal for maintenance and repairs; it defines requirements for maintenance and repair costs of selected sections.

Decision-making processes of both levels of the PMS use the surface properties and bearing capacity of the pavement as neces- 
sary input parameters (most of them are described in the previous part of paper).

Two decision-making procedures could be used generally, priority and optimization, respectively. The former determines technology for pavement rehabilitation according to the current state of pavement serviceability and bearing capacity characteristics.

\subsection{Priority procedure}

The procedure uses results of evaluation for the pavement serviceability characteristics (evenness, skid resistance, surface failures) but the main criterion is the strengthening layer thickness (overlay) calculated for required residual life of the pavement.

If the strengthening thickness $\left(h_{z}\right)$ is not more than $30 \mathrm{~mm}$ a proposal of rehabilitation technology takes into account evaluation of the pavement surface state and pavement unevenness and one of pavement maintenance technologies is proposed (surface dressing, emulsion micro carpet, thin asphalt layer etc.).

If the bearing capacity of pavement is not sufficient for required residual life of the pavement $\left(h_{z}>30 \mathrm{~mm}\right)$ two cases are possible. A total pavement reconstruction is proposed $\left(h_{z}>100\right.$ $\mathrm{mm}$ ) or if the calculated $\mathrm{hz}$ varies between $40 \mathrm{~mm}$ and $100 \mathrm{~mm}$ then the final value of hz depends on the pavement surface state classification according to failures and transversal unevenness.

When possible rehabilitation technologies are known an economic analysis has to be carried out. The special software SEH PS [5] analyses an economic effectiveness of the proposed rehabili- tation technologies. The SEH PS calculates the Internal Rate of Return (IRR) on the base of construction costs of rehabilitation technology and user costs on the base of the pavement surface properties (traffic loading, structural parameters of the road, cost for repairs and maintenance of vehicles, tire wearing, fuel and lubricants costs, lifetime of technology, and other transport costs).

\subsubsection{Optimizing procedure}

The principal point of optimizing procedure is a determination of optimal time for pavement strengthening. Technology of rehabilitation is proposed in relation to time interval between pavement diagnostic and optimal time for pavement strengthening and current state of pavement serviceability.

An optimizing process of rehabilitation time needs, apart from the above described parameters the degradation models of all the parameters. The degradation models are derived from long time measurements on experimental sections and in laboratories. The quality of the model is a basic assumption of optimizing process precision.

\section{Conclusions}

The article describes the principles of the decision making processes in frame of the Slovak PMS. The single parameters of the pavement serviceability and the pavement efficiency are permanently observed and evaluated. The results will use for next specification of the decision criteria that are used for determination of the maintenance and rehabilitation methodology.

\section{References}

[1] CELKO, J., KOMACKA, J.: Analysis of the Pavement Bearing Capacity on the Deflection Bowl Basis, Proc. of 5th Intern. Conference on the Bearing Capacity of Roads and Airfields, Trondheim, 1998, vol. I, pp. 609-617

[2] CELKO, J. et al: Surface Properties of the Pavements (in Slovak), Scientific monograph, EDIS Zilina, 2000, ISBN 80-7100-774-9

[3] KROPAC, O., MUCKA, P.: Relations Between Characteristics of Longitudinal Unevenness of Roads: a review, Strojnicky casopis, $1 /$ 2003, ISSN 0039-2472, pp.49-64.

[4] KROPAC, O., SPRINC, J., SPRINC, M.: Using of Results of International Harmonisation Experiment EVEN - FILTER: longitudinal unevenness of pavements (in Czech), Silnicni obzor, 2/2003, ISSN 0322-7154, pp. 37-48

[5] MIKOLAJ, J., VALUCH, M.: Updated and Extended Program SHE PS (in Slovak), Proc. of the $8^{\text {th }}$ intern. Conference Q 2006 Road Conference, 2006, Rajecke Teplice, pp. 82-87, ISBN 80-85348-71-3

[6] MUCKA, P., DECKY M.: Dynamic Model of Single-Wheel Vehicle for Measuring of Longitudinal Pavement Unevenness (in Slovak), Stavebni obzor, 2/2002, ISSN 210-4027, pp. 59-64

[7] PROCHAZKA, M., SPRINC, J., KROPAC, O.: Theoretical Base for Classification of Pavement Unevenness (in Czech), Silnicni obzor, 7/1980, ISSN 0322-7154

[8] SAYERS, M. W., GILLESPIE, T. D., QUEIROZ, C.: The International Road Roughness Experiment (IRRE): Establishing Correlation and Calibration Standard for Measurements, World Bank, Techn. Paper, No. 45

[9] TP 02/2006 - Measuring and Evaluation of Asphalt Pavement Bearing Capacity with FWD KUAB (in Slovak), Technical regulation, MTPT SR, 2006

[10] TP 10/2006 Pavement Management System (in Slovak), Technical regulation, MTPT SR, 2006

[11] World Road Association: International Experiment to Harmonize Longitudinal and Transverse Profile Measurement and Reporting Procedure, PIARC technical Committee on Surface Characteristics, 2002. 